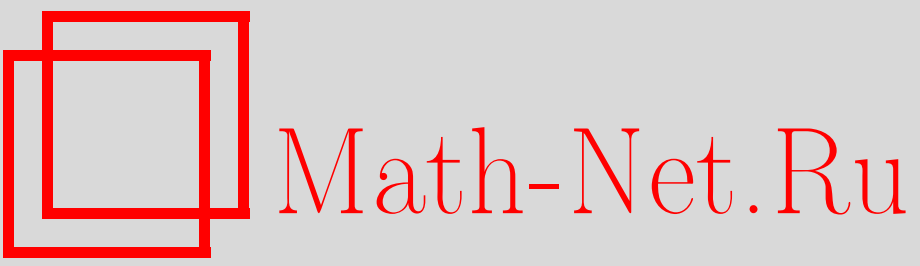

С. Ю. Александров, Лоренц-ковариантная петлевая квантовая гравитация, TMФ, 2004, том 139, номер 3, 363-380

DOI: https://doi.org/10.4213/tmf62

Использование Общероссийского математического портала Math-Net.Ru подразумевает, что вы прочитали и согласны с пользовательским соглашением

http://www . mathnet.ru/rus/agreement

Параметры загрузки:

IP : 44.207 .124 .84

26 апреля 2023 г., 10:54:05 
ТЕОРЕТИЧЕСКАЯ

И МАТЕМАТИЧЕСКАЯ

ФИЗИКА

Том 139, № 3

июнь, 2004

(C) 2004 r.

С. Ю. Александров*

\section{ЛОРЕНЦ-КОВАРИАНТНАЯ ПЕТЛЕВАЯ КВАНТОВАЯ ГРАВИТАЦИЯ}

Представлен обзор лоренц-ковариантного подхода к петлевой квантовой гравитации. Этот подход дает решение проблемы параметра Иммирци, возникающей в стандартном петлевом подходе, основанном на $\mathrm{SU}(2)$-калибровочной группе. Показано, что существует единственное петлевое квантование, сохраняющее все классические симметрии на квантовом уровне, и результаты, полученные в его рамках, такие как спектр оператора плошади, не зависят от параметра Иммирци. Стандартный SU(2)подход нарушает диффеоморфную инвариантность и потому не является корректным квантованием гравитации.

Ключевые слова: петлевая гравитация, каноническое квантование, функциональный интеграл БРСТ.

\section{1. ВВЕДЕНИЕ}

Построение согласованной теории квантовой гравитации представляет на сегодняшний день одну из основных задач, стоящих перед теоретической физикой. Сушествует несколько подходов к квантованию гравитации. Особое место принадлежит теории суперструн и различным суперсимметричным теориям. В них обеспечивается возможность объединения гравитации с полями материи, но при этом приходится явно вводить фоновое пространство, на котором определена теория. Фоновая же независимость должна быть ключевым свойством, выполнение которого требуется от всякой фундаментальной теории, включающей гравитацию.

В качестве альтернативы теории суперструн (или, скорее, как ее дополнение) выступают методы, основанные на каноническом квантовании. Именно здесь проявляются такие характерные черты гравитации как диффеоморфная инвариантность, проблема выбора времени, нелинейность и т.д. Наиболее значительные успехи в этом направлении были достигнуты в рамках петлевой квантовой гравитации [1], [2]. Используя этот подход, удалось построить гильбертово пространство теории, т.е. развить ее на кинематическом уровне, а также получить некоторые физические результаты. Наиболее существенные из них касаются описания геометрических операторов и черных дыр: были

*Institute for Theoretical Physics \& Spinoza Institute, Utrecht University, Postbus 80.195, 3508 TD Utrecht, The Netherlands. E-mail: S.Alexandrov@phys.uu.nl 
рассчитаны спектры операторов площади [3] и объема [4], которые оказались дискретными, а также была воспроизведена формула Бекенштейна-Хокинга для энтропии черной дыры [5].

Основным математическим элементом петлевого подхода является формулировка гравитации в терминах новых переменных - триады и su(2)-связности Аштекара-Барберо [6]. Последняя является вещественным аналогом самодуальной связности, введение которой Аштекаром вызвало целый бум исследований в области канонического квантования гравитации, так как в терминах этой связности связи теории приобретают очень простой вид, становясь полиномиальными [7]. На самом деле есть целое семейство связностей Аштекара-Барберо, параметризуемое параметром $\beta$, который называется параметром Иммирци [8]. Все эти связности на классическом уровне связаны между собой каноническими преобразованиями, поэтому физические результаты в рамках этого формализма не должны зависеть от этого параметра. Но оказывается, что упоминавшиеся выше спектры геометрических операторов, так же как и энтропия черной дыры, прямо пропорционально зависят от параметра $\beta$ [8]. В результате встает вопрос о происхождении этой зависимости, о правомочности полученных результатов и о справедливости всего подхода. Эта проблема носит название проблемы параметра Иммирци, которая до сих пор оставалась нерешенной.

Еще одним слабым местом петлевой гравитации является использование частичной фиксации калибровки до квантования. Исходной калибровочной группой гравитации, действуюшей в касательном пространстве, в используемом формализме первого порядка является группа Лоренца. В стандартном петлевом подходе она редуцируется до группы $\mathrm{SU}(2)$. Это позволяет использовать компактность оставшейся группы и сушественно упрощает каноническую структуру теории. Однако правомочность этой процедуры далеко не очевидна.

В настоящей работе дается обзор результатов, полученных в рамках лоренц-ковариантного формализма, развитого в работах [9]-[13]. Этот формализм позволяет решить сразу обе вышеупомянутые проблемы петлевой гравитации, а также ведет к новым неожиданным результатам.

В разделе 2 дан краткий обзор петлевой гравитации. В разделе 3 строится лоренцковариантный канонический формализм, являюшийся основой для дальнейшего квантования теории. В разделе 4 определен функциональньй интеграл для квантовой гравитации и показывается, что он не зависит от параметра Иммирци $\beta$ [9]. В разделе 5 построенный классический формализм используется для петлевого квантования гравитации. Главный объект исследования - оператор площади пространственноподобной поверхности. Показано, что сушествует двухпараметрическое семейство лоренцевых связностей, диагонализующих оператор площади. Однако только одна из них преобразуется как связность при временнь́х диффеоморфизмах. Поэтому квантование должно быть основано именно на этой связности. При этом соответствующий спектр площади оказывается не зависящим от параметра Иммирци [10], [11]. Более того, доказывается, что стандартный петлевой подход, используюший калибровочную группу $\mathrm{SU}(2)$, неправилен, так как он может быть воспроизведен из ковариантного квантования, где видно, что при этом нарушается диффеоморфная инвариантность, а проблема парамет- 
ра Иммирци есть следствие этого нарушения. В заключении сформулированы основные полученные результаты и намечены дальнейшие перспективы.

\section{2. ОБЗОР ПЕТЛЕВОЙ ГРАВИТАЦИИ}

Основная идея, лежашая в основе петлевого подхода к квантованию гравитации, состоит в том, что физические возбуждения пространства на планковских масштабах одномерны. Другими словами, они должны концентрироваться на одномерных структуpax, таких как линии или графы. Это, безусловно, является предположением и как всякая гипотеза требует своего обоснования. К сожалению, из-за отсутствия каких-либо экспериментальных данных о квантовой гравитации мы можем привести только математические аргументы.

Во-первых, так как фоновая независимость является ключевым свойством для любой теории, включаюшей гравитацию, естественно попытаться определить квантовую теорию в терминах переменных, не зависяших от фона. В формализме первого порядка естественный выбор таких переменных - это голономии связности (вильсоновские линии). В них связность ассоциируется с одномерной кривой, и потому соответствующее квантовое возбуждение тоже будет одномерным. Второе указание на то, что голономии могут описывать фундаментальные степени свободы гравитации, следует из результата, что голономии связности для самонепересекающихся петель являются решениями уравнения Уилера-Девитта. Таким образом, они решают все связи теории и поэтому могут описьвать некоторые физические состояния.

Однако известно, что квантование, основанное на таких петлевых переменных, не работает в случае теории Янга-Миллса. В этом случае физическое состояние, чтобы иметь конечную норму, должно быть распределено по трехмерной области и вильсоновские линии оказываются слишком сингулярными объектами. Но между теорией Янга-Миллса и гравитацией сушествует одно серьезное различие - диффеоморфная инвариантность. Она действует таким образом, что “усредняет” одномерные возбуждения по трехмерной области, приводя в результате к жизнеспособной теории.

Конкретная реализация этих идей осуществляется следующим образом [2]. Исходным пунктом является действие Гильберта-Палатини для гравитации в формализме первого порядка

$$
S_{\mathrm{HP}}=\frac{1}{2} \int \varepsilon_{K L M N} e^{K} \wedge e^{L} \wedge \Omega^{M N}
$$

Здесь $\Omega^{M N}=d \omega^{M N}+\omega^{M}{ }_{K} \wedge \omega^{K N}$, где $\omega$ и $e-1$-формы спин-связности и тетрады, соответственно. Сигнатура метрики выбрана следующим образом: $(-,+,+,+) . K, L, \ldots-$ плоские so(3,1)-индексы в касательном пространстве. Нормировочное условие для тензора Леви-Чивита $\varepsilon_{0123}=1$. Наконец, действие оператора Ходжа определяется как $\star \omega^{K L}=\varepsilon^{K L}{ }_{M N} \omega^{M N} / 2$.

Так как петлевая гравитация основывается на каноническом квантовании, то первый шаг заключается в построении гамильтонова формализма для действия (1). Для этого в стандартном подходе начинают с частичной фиксации калибровки, которая называется в данном случае “временно́й калибровкой”. Она фиксирует направление нормали 
к трехмерным пространственноподобным гиперповерхностям $\{t=$ const $\}$ вдоль направления оси времени $t$. Тем самым калибровочная группа в касательном пространстве редуцируется от $\mathrm{SO}(3,1)$ к $\mathrm{SO}(3)$. Несмотря на кажушуюся безобидность этой процедуры, как будет показано, она имеет далеко идущие последствия.

Тем не менее если использовать “временну́ю” калибровку, то соответствующий канонический формализм принимает вид теории, в которой в качестве канонических переменных выступают взвешенная триада $\widetilde{E}_{a}^{i}$ и внешняя кривизна $K_{i}^{a}$, а также есть 7 связей первого рода, описываюших диффеоморфизмы и локальные $\mathrm{SO}(3)$-вращения [14]. В наших обозначениях $i, j, \ldots$ - пространственные индексы, а $a, b, \ldots$ - плоские so(3)-индексы. Как было замечено Барберо [6], если ввести новые переменные

$$
A_{i}^{(\beta) a}=\Gamma_{i}^{a}(\widetilde{E})-\beta K_{i}^{a}, \quad \widetilde{E}_{(\beta) a}^{i}=-\frac{1}{\beta} \widetilde{E}_{a}^{i}
$$

где $\Gamma_{i}^{a}$ - связность Кристоффеля, построенная из триады $\widetilde{E}_{a}^{i}$, то замена переменных (2) является канонической. Это означает, что скобки Пуассона в новых переменных имеют стандартньй вид

$$
\left\{A_{i}^{(\beta) a}(x), \widetilde{E}_{(\beta) b}^{j}(y)\right\}=\delta_{i}^{j} \delta_{b}^{a} \delta^{3}(x, y) .
$$

Действие гравитации, как всегда, является суммой кинетического члена и связей,

где

$$
\begin{aligned}
S_{A B} & =2 \int d t d^{3} x\left(\widetilde{E}_{(\beta) a}^{i} \partial_{t} A_{i}^{(\beta) a}+N^{a} \mathcal{G}_{a}+N^{i} H_{i}+\underset{\sim}{N H}\right), \\
\mathcal{G}_{a} & =\nabla_{i} \widetilde{E}_{(\beta) a}^{i}=\partial_{i} \widetilde{E}_{(\beta) a}^{i}-\varepsilon_{a b}{ }^{c} A_{i}^{(\beta) b} \widetilde{E}_{(\beta) c}^{i}, \\
H_{i} & =-\widetilde{E}_{(\beta) a}^{k} F_{i k}^{(\beta) a}, \\
H & =-\frac{1}{2} \widetilde{E}_{(\beta) a}^{i} \widetilde{E}_{(\beta) b}^{j}\left(\varepsilon^{a b}{ }_{c} F_{i j}^{(\beta) c}-\left(1+\beta^{2}\right) K_{[i}^{a} K_{j]}^{b}\right),
\end{aligned}
$$

$$
F_{i j}^{(\beta) a}=\partial_{i} A_{j}^{(\beta) a}-\partial_{j} A_{i}^{(\beta) a}-\varepsilon^{a}{ }_{b c} A_{i}^{(\beta) b} A_{j}^{(\beta) c} .
$$

Из явного вида связи $\mathcal{G}_{a}$ и коммутационных соотношений $(3)$ следует, что переменная $A_{i}^{(\beta) a}$ преобразуется как so(3)-связность. Фактически мы имеем целое семейство so(3)связностей, зависяших от параметра $\beta$, который называется параметром Иммирци. Для каждого значения параметра имеется свой набор переменных и связей, но все они связаны каноническим преобразованием [15]. Поэтому на классическом уровне - это фиктивный параметр, от которого никакие результаты не зависят.

Если $\beta= \pm i$, то все связи теории становятся полиномиальными и мы получаем гравитацию в формулировке Аштекара [7]. Однако тогда связность $A_{i}^{(i) a}$ становится комплексной, и необходимо налагать некоторые условия вещественности, чтобы обеспечить эквивалентность с общей теорией относительности [16]. Поэтому петлевая гравитация основана на формулировке с вешественным параметром Иммирци $\beta$. Несмотря на неполиномиальность гамильтоновой связи $H$, она содержит все необходимое для петлевого квантования. Главным при этом является то, что одна из канонических переменных - связность калибровочной групшы симметрии. Это позволяет ввести голономии этой связности

$$
U_{\alpha}\left[A^{(\beta)}\right]=\mathcal{P} \exp \left(\int_{\alpha} d x^{i} A_{i}^{(\beta) a} T_{a}\right),
$$


где $\alpha$ - некоторый путь (замкнутый или открытый), $T_{a}-\operatorname{so}(3)$-генераторы. Если $\alpha-$ замкнутая петля, то можно определить функционал связности как след голономии вокруг этой петли,

$$
\Psi_{\alpha}\left(A^{(\beta)}\right)=\operatorname{Tr} U_{\alpha}\left[A^{(\beta)}\right]
$$

Эти голономии порождают так называемые петлевые состояния, от которых и произошло название петлевой гравитации. Основная идея петлевого квантования состоит в том, что состояния (7) образуют базис физических состояний квантовой гравитации [1]. Математически это означает, что все такие состояния обладают конечной нормой.

Этому утверждению можно придать конкретный смысл, если определить структуру гильбертова пространства, т.е. скалярное произведение на пространстве функционалов от связности [17]. В построенном пространстве можно выделить ортонормированный базис, который дается так называемыми spin network (SN) состояниями [18]. $\mathrm{SN-cocтояние} \mathrm{-} \mathrm{это} \mathrm{функционал} \mathrm{от} \mathrm{связности,} \mathrm{построенный} \mathrm{на} \mathrm{графе,} \mathrm{с} \mathrm{ребрами} \mathrm{кото-}$ рого ассоциированы неприводимые представления калибровочной группы, а с вершинами - базисные элементы в тензорном произведении пространств представлений, соответствующих тем ребрам, которые встречаются в данной вершине. Таким образом, SN это набор из графа, представлений и базисных элементов (переплетающих операторов): $S=\left(\Gamma,\left\{j_{i}\right\},\left\{N_{v}\right\}\right)$. Если $N_{v}$ - инвариантные тензоры, принадлежащие тривиальному представлению, то мы получаем калибровочно-инвариантный SN. В свою очередь, диффеоморфно-инвариантные состояния можно получить, если ограничиться только топологическими свойствами графа, определяюшего SN, т.е. отбросить конкретную координатную реализацию вложения графа в пространство. Результат этой факторизации кинематическое гильбертово пространство в петлевом подходе.

На построенном гильбертовом пространстве можно определить квантовые операторы, соответствующие различным классическим величинам, в частности, геометрические операторы плошади [3] и объема [4]. Основным физическим результатом, полученным в рамках петлевой гравитации, является вычисление спектров этих операторов. Оба они оказываются дискретными, что интерпретируется как существование в квантовой гравитации минимальной длины и дискретной структуры пространства-времени. Мы приведем лишь результат для основной серии собственных значений оператора площади.

Пусть $\left\{p_{k}\right\}=\Sigma \cap \Gamma$, пересечение измеряемой двумерной поверхности $\Sigma$ с графом $\Gamma$, задаюшим $\mathrm{SN}$, не содержит вершин графа и каждая точка пересечения $p_{k}$ характеризуется неприводимым представлением $\mathrm{SU}(2) j_{k}$, которое несет ребро граф̆а, содержащее эту точку. Тогда любое SN-состояние является собственным состоянием оператора площади и спектр собственных значений дается спектром оператора Казимира группы $\mathrm{SU}(2)$,

$$
\mathcal{S}(\Sigma)=8 \pi \beta \hbar G \sum_{k} \sqrt{j_{k}\left(j_{k}+1\right)}
$$

Этот результат был использован в различных приложениях теории. В частности, с его помошью была воспроизведена формула Бекенштейна-Хокинга для энтропии черной 
дыры, принимаюшая следуюший вид [5]:

$$
S_{\mathrm{BH}}=\frac{\beta_{0}}{4 \beta \hbar G} A_{\mathrm{hor}}, \quad \beta_{0}=\frac{\ln 2}{\pi \sqrt{3}} .
$$

Однако, как было впервые замечено Иммирци [8], в спектр плошади явно входит параметр $\beta$, который на классическом уровне являлся нефизическим параметром в каноническом преобразовании переменных. В связи с этим было выдвинуто предположение [19], что квантования общей теории относительности при разных значениях $\beta$ физически неэквивалентны. Таким образом, этот параметр должен рассматриваться как новая фундаментальная постоянная. Было предложено фиксировать значение $\beta$ условием, чтобы коэффициент перед плошадью горизонта в формуле (9) равнялся $1 / 4$, как в стандартной формуле Бекенштейна-Хокинга. Тогда необходимо положить $\beta=\beta_{0}$. Однако эта гипотеза не разрешает проблему происхождения этой зависимости, которая остается мистической в стандартном подходе. Как будет показано ниже, она получает естественное объяснение в лоренц-ковариантном петлевом квантовании.

\section{3. ЛОРЕНЦ-КОВАРИАНТНАЯ КАНОНИЧЕСКАЯ ФОРМУЛИРОВКА ГРАВИТАЦИИ}

Наша первая цель - построить каноническую формулировку гравитации с $\mathrm{SO}(3,1)$-калибровочной группой, в которой одной из канонических переменных была бы связность [9]. Естественная стартовая точка - действие Гильберта-Палатини (1). Однако мы хотим с самого начала ввести в теорию параметр Иммирци. Это может быть сделано ковариантным образом, если рассмотреть обобшенное действие Гильберта-Палатини, впервые предложенное Хольстом,

$$
S_{(\beta)}=\frac{1}{2} \int \varepsilon_{K L M N} e^{M} \wedge e^{L} \wedge\left(\Omega^{M N}+\frac{1}{\beta} \star \Omega^{M N}\right) .
$$

Фактически оно эквивалентно обычному действию (1), так как из него следуют те же уравнения движения. В работе [20] было показано, что во "временно́й" калибровке это действие воспроизводит каноническую формулировку гравитации Аштекара-Барберо (4), а параметр $\beta$ совпадает с параметром Иммирци. Мы построим канонический формализм для теории, описываемой действием (10), не налагая никакой калибровки.

Разобъем координаты $x^{\mu}$ на "временну́ю" $t$ и “пространственные" $x^{i}$ компоненты и введем обозначения

$$
e^{0}=N d t+\chi_{a} E_{i}^{a} d x^{i}, \quad e^{a}=E_{i}^{a} d x^{i}+E_{i}^{a} N^{i} d t .
$$

Соотношение (11) описывает самое обшее $3+1$ разложение тетрады. $E_{i}^{a}$ - обычное поле триады, тогда как $\chi_{a}$ описывает отклонение нормали к гиперповерхностям $\{t=\mathrm{const}\}$ от временно́го направления. В стандартном подходе это поле полагается равным нулю, что соответствует выбору “временно́й” калибровки.

Обозначим через $E_{a}^{i}$ матрицу, обратную к $E_{i}^{a}$, и введем взвешенные поля: $\widetilde{E}_{a}^{i}=$ $\sqrt{h} E_{a}^{i}, \underset{\sim}{N}=(\sqrt{h})^{-1} N$, где $\sqrt{h}=\operatorname{det} E_{i}^{a}$. Кроме того, сделаем замену переменных

$$
N^{i}=\mathcal{N}_{\mathcal{D}}^{i}+\widetilde{E}_{a}^{i} \chi^{a} \underset{\sim}{\mathcal{N}}, \quad \underset{\sim}{N}=\underset{\sim}{\mathcal{N}}+\underset{\sim}{E_{i}^{a}} \chi_{a} \mathcal{N}_{\mathcal{D}}^{i}
$$


и введем so(3,1)-мультиплеты, объединив пару so(3)-индексов в один so(3,1)-индекс:

$$
\begin{aligned}
A_{i}^{X} & =\left(\omega_{i}^{0 a}, \frac{1}{2} \varepsilon^{a}{ }_{b c} \omega_{i}^{b c}\right)-\text { мультиплет связности, } \\
\widetilde{P}_{X}^{i} & =\left(\widetilde{E}_{a}^{i}, \varepsilon_{a}{ }^{b c} \widetilde{E}_{b}^{i} \chi_{c}\right)-\text { первый триадный мультиплет, } \\
\widetilde{Q}_{X}^{i} & =\left(-\varepsilon_{a}{ }^{b c} \widetilde{E}_{b}^{i} \chi_{c}, \widetilde{E}_{a}^{i}\right)-\text { второй триадный мультиплет, } \\
\widetilde{P}_{(\beta) X}{ }^{i} & =\widetilde{P}_{X}^{i}-\frac{1}{\beta} \widetilde{Q}_{X}^{i} \quad-\text { канонический триадный мультиплет. }
\end{aligned}
$$

Различные соотношения между триадными мультиплетами представлены в приложении А. Оказывается, что это мультиплеты в присоединенном представлении алгебры Лоренца со структурными константами $f_{X Y}^{Z}$. В работе [9] было показано, что действие (10) может быть представлено в виде

$$
S_{(\beta)}=\int d t d^{3} x\left(\widetilde{P}_{(\beta) X}{ }^{i} \partial_{t} A_{i}^{X}+A_{0}^{X} \mathcal{G}_{X}+\mathcal{N}_{\mathcal{D}}^{i} \mathcal{H}_{i}+\underset{\sim}{\mathcal{N}} \mathcal{H}\right)
$$

где

$$
\begin{aligned}
\mathcal{G}_{X} & =\partial_{i} \widetilde{P}_{(\beta) X}{ }^{i}+f_{X Y}^{Z} A_{i}^{Y} \widetilde{P}_{(\beta) Z}{ }^{i}, \\
\mathcal{H}_{i} & =-\widetilde{P}_{(\beta) X^{j} F_{i j}^{X},} \\
\mathcal{H} & =-\frac{1}{2\left(1+\beta^{-2}\right)} \widetilde{P}_{(\beta) X}{ }^{i} \widetilde{P}_{(\beta) Y}{ }^{j} f_{Z}^{X Y} R_{W}^{Z} F_{i j}^{W}, \\
F_{i j}^{X} & =\partial_{i} A_{j}^{X}-\partial_{j} A_{i}^{X}+f_{Y Z}^{X} A_{i}^{Y} A_{j}^{Z} .
\end{aligned}
$$

Здесь поднимание и опускание шестимерных индексов производится с помошью формы Киллинга

$$
g_{X Y}=\frac{1}{4} f_{X Z_{1}}^{Z_{2}} f_{Y Z_{2}}^{Z_{1}}, \quad g^{X Y}=\left(g^{-1}\right)^{X Y}, \quad g_{X Y}=\left(\begin{array}{cc}
\delta_{a b} & 0 \\
0 & -\delta_{a b}
\end{array}\right) .
$$

Матрицы $R_{X}^{Y}$ и $\Pi_{X}^{Y}$, появляюшиеся в дальнейшем, введены в приложении А.

Каноническими переменными являются $A_{i}^{X}$ и $\widetilde{P}_{(\beta) X}{ }^{i}$, .e. $^{1)}$

$$
\left\{A_{i}^{X}, \widetilde{P}_{(\beta) Y}^{j}\right\}=\delta_{i}^{j} \delta_{Y}^{X} .
$$

Однако, как следует из определения $(13)$, не все импульсы $\widetilde{P}_{(\beta)} X^{i}$ являются независимыми. Между ними существует 6 связей, которые также порождают 6 вторичных связей. Все они могут быть записаны в ковариантной форме

$$
\begin{aligned}
\phi^{i j} & =\Pi^{X Y} \widetilde{Q}_{X}^{i} \widetilde{Q}_{Y}^{j}, \\
\psi^{i j} & =f^{X Y Z} \widetilde{Q}_{X}^{[l} \widetilde{Q}_{Y}^{\{j]} \partial_{l} \widetilde{Q}_{Z}^{i\}}-2(\widetilde{Q} \widetilde{Q})^{\{i[j\}} \widetilde{Q}_{Z}^{l]} A_{l}^{Z},
\end{aligned}
$$

\footnotetext{
1) Мы не будем указывать явно зависимость от пространственных координат в коммутационных соотношениях. Всегда подразумевается, что результат содержит $\delta$-функцию от этих координат.
} 
где $(\widetilde{Q} \widetilde{Q})^{i j}=g^{X Y} \widetilde{Q}_{X}^{i} \widetilde{Q}_{Y}^{j}$. Эти связи являются связями второго рода с матрицей коммутаторов

$$
\begin{aligned}
& \Delta=\left(\begin{array}{cc}
0 & D_{1} \\
-D_{1} & D_{2}
\end{array}\right), \quad D_{1}^{(i j)(k l)}=\left\{\phi^{i j}, \psi^{k l}\right\}=\frac{4 \beta^{2}}{1+\beta^{2}}(\widetilde{Q} \widetilde{Q})^{\{i[j\}}(\widetilde{Q} \widetilde{Q})^{\{k] l\}}, \\
& D_{2}^{(i j)(k l)}=\left\{\psi^{i j}, \psi^{k l}\right\} \neq 0 \text {. }
\end{aligned}
$$

Кроме того, имеется 10 связей первого рода $\Phi_{\alpha}=\left(\mathcal{G}_{X}, \mathcal{D}_{i}, \mathcal{H}\right)$, генерирующих so $(3,1)$-калибровочные преобразования и диффеоморфизмы. Мы ввели

$$
\mathcal{D}_{i}=\mathcal{H}_{i}+A_{i}^{X} \mathcal{G}_{X}=\partial_{j}\left(A_{i}^{X} \widetilde{P}_{(\beta) X}{ }^{j}\right)-\widetilde{P}_{(\beta) X}{ }^{j} \partial_{i} A_{j}^{X} .
$$

Алгебра связей $\Phi_{\alpha}$ представлена в приложении Б.

Связи второго рода $\varphi_{r}=\left(\phi^{i j}, \psi^{i j}\right)$ приводят к необходимости ввести скобку Дирака

$$
\{K, L\}_{\mathrm{D}}=\{K, L\}-\left\{K, \varphi_{r}\right\}\left(\Delta^{-1}\right)^{r r^{\prime}}\left\{\varphi_{r^{\prime}}, L\right\} .
$$

Из-за треугольности матрицы $\Delta$ (19) скобка Дирака обладает следуюшим важным свойством: если одна из функций совпадает со связью первого рода, то скобка Дирака совпадает со скобкой Пуассона (кроме случая, когда $K$ - гамильтонова связь, а $L$ зависит от связности).

Используя этот факт, можно найти законы преобразования мультиплетов:

$$
\begin{aligned}
\left\{\mathcal{G}_{X}, A_{i}^{Y}\right\}_{\mathrm{D}} & =\delta_{X}^{Y} \partial_{i}-f_{X Z}^{Y} A_{i}^{Z}, \\
\left\{\mathcal{G}_{X}, \widetilde{P}_{Y}^{i}\right\}_{\mathrm{D}} & =f_{X Y}^{Z} \widetilde{P}_{Z}^{i}, \\
\left\{\mathcal{G}_{X}, \widetilde{Q}_{Y}^{i}\right\}_{\mathrm{D}} & =f_{X Y}^{Z} \widetilde{Q}_{Z}^{i} .
\end{aligned}
$$

Как и должно быть, триадные мультиплеты образуют присоединенное представление so $(3,1)$, тогда как $A_{i}^{X}$ - истинная лоренцева связность.

Наконец, можно найти скобки Дирака канонических переменных [9]:

$$
\begin{aligned}
\left\{\widetilde{P}_{(\beta) X}{ }^{i}, \widetilde{P}_{(\beta) Y}{ }^{j}\right\}_{\mathrm{D}} & =0, \\
\left\{A_{i}^{X}, \widetilde{P}_{(\beta) Y}\right\}_{\mathrm{D}} & =\delta_{i}^{j} \delta_{Y}^{X}-\frac{1}{2} R^{X Z}\left(\widetilde{Q}_{Z}^{j}{\underset{\sim}{W}}_{i}^{W}+\delta_{i}^{j} I_{(Q) Z}^{W}\right) g_{W Y}, \\
\left\{A_{i}^{X}, A_{j}^{Y}\right\}_{\mathrm{D}} & \neq 0 .
\end{aligned}
$$

Здесь введены так называемые обратные триадные мультиплеты $\underset{\sim}{P} \underset{i}{X}$ и $\underset{\sim}{Q_{i}^{X}}$, а также матрицы $I_{(P) X}^{Y}=\widetilde{P}_{X}^{i} P_{i}^{Y}$ и $I_{(Q) X}^{Y}=\widetilde{Q}_{X}^{i} \underset{\sim}{Q_{i}^{Y}}$, которые являются проекторами на $\widetilde{P}$ - и $\widetilde{Q}$-мультиплеты в линейном пространстве, составленном из этих векторов.

Коммутаторы (23) будут служить исходной точкой для канонического квантования теории. Главная особенность соотношений (23) - некоммутативность связности, что является сушественным отличием от гравитации Аштекара-Барберо. Другая характерная черта, которая будет иметь важные следствия, - это недиагональность коммутатора

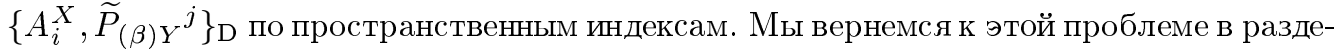
ле 5 , когда будем обсуждать оператор плошади в ковариантном формализме. 


\section{4. КВАНТОВАНИЕ МЕТОДОМ ФУНКЦИОНАЛЬНЫХ ИНТЕГРАЛОВ}

Гравитация - это динамическая система со связями. При этом в используемой нами формулировке (14) алгебра связей оказывается открытой, т.е. структурные константы, определяемые соотношениями (Б.1) (см. приложение Б), являются функциями канонических координат (в этом смысле они больше не являются "константами"; тем не менее мы будем использовать этот стандартный термин). В этом случае, чтобы построить функциональный интеграл, описывающий теорию, необходимо использовать процедуру BRST-квантования (Бекки-Руэ-Стора-Тютина), развитую для гамильтоновых систем с открытой алгеброй связей [21], [22]. В итоге BRST-анализ действия (14) приводит к следующему функциональному интегралу [9]:

$$
\begin{aligned}
Z[j, J]= & \int \mathcal{D} A_{i}^{X} \mathcal{D} \widetilde{P}_{(\beta) X}{ }^{i} \mathcal{D N} \mathcal{N}^{\alpha} \mathcal{D} c^{\alpha} \mathcal{D} \bar{c}_{\alpha} \sqrt{|\Delta|} \delta\left(\phi^{i j}\right) \delta\left(\psi^{i j}\right) \delta\left(f^{\alpha}+g^{\alpha}\right) \times \\
& \times \exp \left[i \int d t\left(L_{\mathrm{eff}}+j_{a}^{i} A_{i}^{a}+J_{i}^{a} \widetilde{P}_{a}^{i}\right)\right]
\end{aligned}
$$

где

$$
\begin{aligned}
L_{\mathrm{eff}}= & \widetilde{P}_{(\beta) X}{ }^{i} \partial_{t} A_{i}^{X}+\mathcal{N}^{\alpha} \Phi_{\alpha}-i \bar{c}_{\beta}\left(\frac{\partial g^{\beta}}{\partial \mathcal{N}^{\alpha}} \partial_{t}-\frac{\partial g^{\beta}}{\partial \mathcal{N}^{\gamma}} C_{\alpha \lambda}^{\gamma} \mathcal{N}^{\lambda}+\left\{\Phi_{\alpha}, f^{\beta}\right\}_{\mathrm{D}}\right) c^{\alpha}- \\
& -\bar{c}_{\xi} \bar{c}_{\eta}\left(\frac{\partial g^{\eta}}{\partial \mathcal{N}^{\delta}}\left\{f^{\xi}, C_{\alpha \beta}^{\delta}\right\}_{\mathrm{D}}+3 \frac{\partial g^{\xi}}{\partial \mathcal{N}^{\delta}} \frac{\partial g^{\eta}}{\partial \mathcal{N}^{\lambda}} U_{\alpha \beta \gamma}^{(2) \delta \lambda} \mathcal{N}^{\gamma}\right) c^{\alpha} c^{\beta}- \\
& -i \bar{c}_{\alpha} \bar{c}_{\xi} \bar{c}_{\eta} \frac{\partial g^{\xi}}{\partial \mathcal{N}^{\lambda}} \frac{\partial g^{\eta}}{\partial \mathcal{N}^{\sigma}}\left\{f^{\alpha}, U_{\beta \gamma \delta}^{(2) \lambda \sigma}\right\}_{\mathrm{D}} c^{\beta} c^{\gamma} c^{\delta}
\end{aligned}
$$

Здесь $c^{\alpha}, \bar{c}_{\alpha}$ - антикоммутируюшие духи, $f^{\alpha}(\widetilde{P}, A)+g^{\alpha}(\mathcal{N})$ - функции, фиксируюшие калибровку, и учтено, что система ограничена связями второго рода $\phi^{i j}$ и $\psi^{i j}$ с матрицей коммутаторов $\Delta$, заданной в (19). Мы видим, что в эффективном действии появляются высшие духовые члены. Их появление связано с тем, что структурные константы зависят от канонических переменных. Это же является причиной появления структурных функций второго порядка $U^{(2)}$. Их ненулевые компоненты заданы соотношением

$$
\begin{aligned}
& c^{0}(x) U_{00^{\prime} 0^{\prime \prime}}^{(2) X Y} c^{0}\left(x^{\prime}\right) c^{0}\left(x^{\prime \prime}\right)= \\
& \quad=-\frac{1}{2\left(1+\beta^{-2}\right)} c^{0} \partial_{i} c^{0} \partial_{k} c^{0} R^{X Z} R^{W Y} \widetilde{Q}_{Z}^{i} \widetilde{Q}_{W}^{k} \delta\left(x, x^{\prime}\right) \delta\left(x^{\prime}, x^{\prime \prime}\right),
\end{aligned}
$$

где индекс 0 соответствует гамильтоновой связи. Заметим, что как в теории Янга-Миллса, так и в комплексной гравитации Аштекара член с $U^{(2)}$ отсутствует в BRST-заряде.

Таким образом, мы приходим к очень сложному функциональному интегралу, содержашему мультидуховые взаимодействия. Однако оказывается, что при подходящем выборе калибровки эти взаимодействия исчезают. Действительно, чтобы устранить члены со структурными функциями второго порядка, достаточно потребовать, чтобы функции $g^{\alpha}$ не зависели от $\mathcal{N}_{\mathcal{G}}^{X}$. Кроме того, если при этом функции $f^{\alpha}$ не зависят от связности $A_{i}^{X}$, пропадает также член со скобкой Дирака $\left\{f^{\xi}, C_{\alpha \beta}^{\delta}\right\}_{\mathrm{D}}$. Таким образом, мы 
ограничиваем возможные типы калибровочных условий теми, которые не фиксируют множители Лагранжа для гауссовой связи и компоненты связности ${ }^{2)}$. Такие калибровки были введены в работе [24] и называются калибровками Янга-Миллса. Для них высшие духовые члены в эффективном действии отсутствуют и функциональный интеграл совпадает с обычным функциональным интегралом для теории типа Янга-Миллса в гамильтоновой формулировке. Он дается выражением (24), где

$$
L_{\text {eff }}=L_{(\beta)}-i \bar{c}_{\beta}\left(\frac{\partial g^{\beta}}{\partial \mathcal{N}^{\alpha}} \partial_{t}-\frac{\partial g^{\beta}}{\partial \mathcal{N} \gamma} C_{\alpha \delta^{\gamma}}^{\gamma} \mathcal{N}^{\delta}+\left\{\Phi_{\alpha}^{(\beta)}, f^{\beta}(\widetilde{P})\right\}_{\mathrm{D}}^{(\beta)}\right) c^{\alpha}
$$

В работе [9] была исследована зависимость функционального интеграла (24) от параметра Иммирци $\beta$ и доказано, что в калибровке Янга-Миллса эта зависимость отсутствует. Таким образом, проблема параметра Иммирци не возникает в данном подходе к квантованию гравитации.

\section{5. КОВАРИАНТНАЯ ПЕТЛЕВАЯ ГРАВИТАЦИЯ И ОПЕРАТОР ПЛОЩАДИ}

5.1. Лоренцева связность и оператор площади. В этом разделе описанная выше лоренш-ковариантная формулировка используется для построения петлевой гравитации. Ключевой факт, который делает это возможным, состоит в том, что одна из переменных $A_{i}^{X}$ является лоренцевой связностью (22), так что можно построить оператор вильсоновской линии

$$
\widehat{U}_{\alpha}[A]=\mathcal{P} \exp \left(\int_{\alpha} d x^{i} A_{i}^{X} T_{X}\right),
$$

где $T_{X}$ - генераторы алгебры so $(3,1)$. Используя эти операторы вместо (6), можно попытаться построить гильбертово пространство, как и в $\mathrm{SU}(2)$-случае. Однако мы сталкиваемся с серьезной трудностью на этом пути, так как вместо простых коммутационных соотношений (3) канонические переменные подчиняются очень нетривиальной алгебре скобок Дирака (23). В частности, операторы типа (28) не образуют петлевую алгебру. Более того, так как связность $A_{i}^{X}$ некоммутативна, не существует соответствующего представления, где бы она была диагональным оператором.

Несмотря на эти трудности, некоторые результаты могут быть получены исходя только из коммутационных соотношений (23). В частности, можно вывести спектр оператора площади, которьй был до этого предметом широкого исследования в рамках стандартного SU(2)-подхода к петлевой гравитации [3]. Чтобы его ввести, определим сначала оператор триады, усредненной по двумерной поверхности, вложенной в трехмерное многообразие:

$$
\widetilde{P}_{X}(\Sigma)=\int_{\Sigma} d^{2} \sigma n_{i}(\sigma) \widetilde{P}_{X}^{i}(\sigma)
$$

\footnotetext{
2) То, что калибровочные условия на компоненты связности приводят к проблемам на уровне функционального интеграла, было замечено ранее при исследовании конечномерной модели гравитации Аштекара [23].
} 
где вложение описывается координатами $x^{i}(\vec{\sigma})$, а $n_{i}=\varepsilon_{i j k}\left(\partial x^{j} / \partial \sigma^{1}\right)\left(\partial x^{k} / \partial \sigma^{2}\right)$ - нормаль к поверхности. Тогда регуляризованный оператор плошади определяется следующим образом:

$$
\mathcal{S}(\Sigma)=\lim _{\rho \rightarrow \infty} \sum_{n} \sqrt{g\left(\Sigma_{n}\right)}, \quad g(\Sigma)=g^{X Y} \widetilde{P}_{X}(\Sigma) \widetilde{P}_{Y}(\Sigma),
$$

где сумма берется по разбиению $\rho$ поверхности $\Sigma$ на малые части $\Sigma_{n}, \bigcup_{n} \Sigma_{n}=\Sigma$. Нетрудно видеть, что в классическом пределе этот оператор переходит в стандартное выражение

$$
\mathcal{S}(\Sigma)=\int_{\Sigma} d^{2} \sigma \sqrt{n_{i} n_{j} g^{i j}}, \quad g^{i j}=g^{X Y} \widetilde{P}_{X}^{i} \widetilde{P}_{Y}^{j}
$$

Спектр оператора площади может быть найден путем изучения действия усредненной триады на состояние, порожденное вильсоновской линией. Легко показать, что оператор плошади (30) недиагонален на состояниях, порожденных вильсоновскими линиями (28) [10]. Причиной этого является то, что коммутатор $\left\{A_{k}^{Y}, \widetilde{P}_{X}^{l}\right\}_{\mathrm{D}}$ не пропорционален $\delta_{k}^{l}$. Однако оператор площади может быть диагонализован, если учесть произвол в определении вильсоновской линии. Этот произвол связан с тем, что соответствующий оператор можно определить для любой величины, являющейся связностью, и она не обязана быть при этом канонической переменной. Поэтому, чтобы найти спектр оператора площади, нужно найти такую связность $\mathcal{A}_{i}^{X}$, для которой вильсоновская линия

$$
U_{\alpha}[\mathcal{A}]=\mathcal{P} \exp \left(\int_{\alpha} d x^{i} \mathcal{A}_{i}^{X} T_{X}\right)
$$

является собственным состоянием этого оператора.

В работе [11] показано, что существует двухпараметрическое семейство лоренцевых связностей $\mathcal{A}_{i}^{X}(a, b)$, удовлетворяюших следуюшим условиям:

$$
\begin{aligned}
\left\{\mathcal{G}(n), \mathcal{A}_{i}^{X}\right\}_{\mathrm{D}} & =\partial_{i} n^{X}-f_{Y}^{X} n^{Y} \mathcal{A}_{i}^{Z}, \\
\left\{\mathcal{D}(\vec{N}), \mathcal{A}_{i}^{X}\right\}_{\mathrm{D}} & =\mathcal{A}_{j}^{X} \partial_{i} N^{j}+N^{j} \partial_{j} \mathcal{A}_{i}^{X}, \\
\left\{\mathcal{A}_{i}^{X}, \widetilde{P}_{Y}^{j}\right\}_{\mathrm{D}} & \sim \delta_{i}^{j} .
\end{aligned}
$$

Эти связности можно представить в терминах исходных переменных

$$
\begin{aligned}
\mathcal{A}_{i}^{X}(a, b)= & A_{i}^{X}+\frac{1}{2}\left(\left(1+\frac{a}{\beta}\right) g^{X X^{\prime}}-\frac{1}{\beta}(1-b) \Pi^{X X^{\prime}}\right) I_{(Q) X^{\prime}}^{T} \frac{R_{T}^{Y}}{1+\beta^{-2}} f_{Y Z}^{W} \underset{\sim}{P_{i}^{Z}} \mathcal{G}_{W}+ \\
& +\left(a \delta_{X^{\prime}}^{X}+b \Pi_{X^{\prime}}^{X}\right)\left(I_{(Q)}^{X^{\prime} W} \Pi_{W Z} A_{i}^{Z}+\Lambda_{i}^{X^{\prime}}\right)
\end{aligned}
$$

где

$$
\Lambda_{i}^{X}=g^{X X^{\prime}} \Pi_{R}^{Z}\left(I_{(Q) X^{\prime}}^{R} f_{Y Z}^{W} \underset{\sim}{Q_{i}^{Y}} \partial_{l} \widetilde{Q}_{W}^{l}+\frac{1}{2} f_{Y Z}^{W} \widetilde{Q}_{X^{\prime}}^{k} \underset{\sim}{Q_{i}^{Y}} \widetilde{Q}_{W}^{l} \partial_{k}{\underset{\sim}{l}}_{l}^{R}\right) .
$$

Можно показать, что в действительности величина $\Lambda$ не зависит от триады $\widetilde{E}$ и является функцией только поля $\chi$ :

$$
\Lambda_{i}^{X}(\chi)=-g^{X Y} \frac{\widetilde{Q}_{Y}^{j} \underset{\sim}{E_{j}^{a} \partial_{i} \chi_{a}}}{1-\chi^{2}}=\left(-\frac{\varepsilon^{a b c} \chi_{b} \partial_{i} \chi_{c}}{1-\chi^{2}}, \frac{\partial_{i} \chi^{a}}{1-\chi^{2}}\right) .
$$


Связности (36) удовлетворяют следуюшим коммутационным соотношениям:

$$
\begin{aligned}
\left\{\mathcal{A}_{i}^{X}(a, b), \widetilde{P}_{Y}^{j}\right\}_{\mathrm{D}} & =\delta_{i}^{j}\left((1-b) \delta_{X^{\prime}}^{X}-a \Pi_{X^{\prime}}^{X}\right) I_{(P) Y}^{X^{\prime}}, \\
\left\{\mathcal{A}_{i}^{X}(a, b),{\underset{\sim}{j}}_{j}^{Y}\right\}_{\mathrm{D}} & =-\left((1-b) \delta_{X^{\prime}}^{X}-a \Pi_{X^{\prime}}^{X}\right) P_{\sim}^{X^{\prime}} P_{\sim}^{Y}, \\
\left\{\mathcal{A}_{i}^{X}(a, b), I_{(P)}^{Y}\right\}_{\mathrm{D}} & =0 .
\end{aligned}
$$

Замечательное свойство этих соотношений заключается в том, что проекторы $I_{(P)}$ и $I_{(Q)}$ ведут себя подобно $c$-числам с точки зрения алгебры коммутаторов. Отсюда следует, что поле $\chi$ коммутирует с любой связностью $\mathcal{A}(a, b)$.

Рассмотрим состояние, порожденное вильсоновской линией (32), определенной с помошью одной из связностей семейства (36). Основываясь на коммутационных соотношениях (39), нетрудно получить действие оператора плошади (30) на такую вильсоновскую линию. Оно описьвается алгебраическим оператором, действующим только на групповые индексы,

$$
\begin{aligned}
\mathcal{S} U_{\alpha}[\mathcal{A}(a, b)] & =\hbar U_{\alpha_{1}}[\mathcal{A}(a, b)] \sqrt{\Delta_{\mathcal{S}}} U_{\alpha_{2}}[\mathcal{A}(a, b)], \\
\Delta_{\mathcal{S}} & =\left(a^{2} I_{(Q)}^{X Y}-(1-b)^{2} I_{(P)}^{X Y}+a(1-b) \Pi^{X Y}\right) T_{X} T_{Y},
\end{aligned}
$$

который может быть выражен через операторы Казимира $\mathrm{SO}(3,1)$ и подгруппы $\mathrm{SO}(3)$, оставляюшей инвариантным вектор $v_{\chi}=\left(1-\chi^{2}\right)^{-1 / 2}\left(1, \chi_{a}\right)$ :

$$
\Delta_{\mathcal{S}}=\left(a^{2}+(1-b)^{2}\right) C(\operatorname{so}(3))-(1-b)^{2} C_{1}(\operatorname{so}(3,1))+a(1-b) C_{2}(\operatorname{so}(3,1)) .
$$

В результате оператор площади оказывается диагонализуемым и его спектр дается собственными значениями операторов Казимира для неприводимых представлений группы Лоренца. Для унитарных представлений спектр принимает вид

$$
\begin{aligned}
\mathcal{S}^{\mathrm{pr}} & \sim \hbar \sqrt{\left(a^{2}+(1-b)^{2}\right) j(j+1)+(1-b)^{2}\left(\rho^{2}-n^{2}+1\right)+2 a(1-b) n \rho}, \\
\mathcal{S}^{\text {sup }} & \sim \hbar \sqrt{\left(a^{2}+(1-b)^{2}\right) j(j+1)+(1-b)^{2}\left(1-\rho^{2}\right)}
\end{aligned}
$$

для основной и дополнительной серий, соответственно. Здесь $2 n \in \mathbf{N}, \rho \in \mathbf{R},(j-n) \in$ $\mathbf{N}$, а в случае дополнительной серии $-1<\rho<1, j \in \mathbf{N}$. Благодаря этим свойствам полученный спектр всегда вешествен. Причина этого может заключаться в появлении оператора Казимира $\mathrm{SO}(3)$-подгруппы в операторе (41). Он сокрашает отрицательный вклад оператора Казимира группы Лоренца, поэтому его присутствие очень сушественно. И действительно, меняя параметры $a$ и $b$, невозможно получить спектр только в виде казимиров группы Лоренца.

5.2. Трансформационные свойства при временнь́х диффеоморфизмах. До сих пор мы имели двухпараметрическое семейство лореншевых связностей (36). Его существование означает наличие произвола в квантовании теории, так как все допустимые связности ведут к разным результатам для спектра оператора плошади (41), т.е. определяют неэквивалентные квантования гравитации. Если мы надеемся найти однозначный ответ в поисках теории квантовой гравитации (по крайней мере в рамках петлевого подхода), мы должны наложить некоторое дополнительное физическое условие, 
которое бы позволило отобрать одну-единственную связность из найденного семейства. Именно эта связность и должна использоваться в определении петлевых квантовых операторов.

Предыдуший анализ был основан на симметриях: мы требовали, чтобы симметрии классической теории сохранялись при квантовании. Это требование было выражено через трансформационные свойства связности, в терминах которой затем определялась вильсоновская линия. Однако среди условий (33) и (34) одна симметрия была до сих пор пропушена, а именно симметрия относительно временнь́х диффеоморфизмов. Поэтому в качестве искомого дополнительного условия мы должны взять требование правильных трансформационных свойств при таких преобразованиях. Только связность, удовлетворяюшая этому требованию, может привести к квантовой теории, не зависяшей от введения пространственноподобной гиперповерхности, так как только в этом случае при временны́х трансляциях вильсоновская линия отображается в такой же оператор, но уже на сдвинутой во времени гиперповерхности. В противном случае четырехмерная алгебра диффеоморфизмов приобретает квантовую аномалию.

Таким образом, мы приходим к необходимости наложить на связность (36) следующее условие:

$$
\begin{aligned}
& \delta_{\left(\xi^{0}\right)} \mathcal{A}_{i}^{X}=\xi^{0} \partial_{0} \mathcal{A}_{i}^{X}+\mathcal{A}_{0}^{X} \partial_{i} \xi^{0}, \\
& \delta_{\left(\xi^{0}\right)} \mathcal{A}_{0}^{X}=\partial_{0}\left(\xi^{0} \mathcal{A}_{0}^{X}\right),
\end{aligned}
$$

где $\mathcal{A}_{0}^{X}$ - соответствуюшее обобшение временно́й компоненты исходной связности $A_{0}^{i}$. Связность, удовлетворяюшую условиям (33), (34) и (44), будем называть пространственно-временной связностью.

В работе [11] было показано, что на поверхности гауссовой связи и уравнений движения семейство (36) содержит только одну лоренцеву пространственно-временную связность:

$$
\mathcal{A}_{i}^{X} \equiv \mathcal{A}_{i}^{X}(0,0)=A_{i}^{X}+\frac{1}{2\left(1+\beta^{-2}\right)} R_{S}^{X} I_{(Q)}^{S T} R_{T}^{Z} f_{Z W}^{Y}{\underset{\sim}{i}}_{i}^{W} \mathcal{G}_{Y}
$$

Таким образом, дополнительное условие (44) оказывается достаточным, чтобы устранить произвол в квантовании, связанный с выбором связности, так как мы получили одну-единственную связность, удовлетворяющую всем необходимым требованиям. Несмотря на то что этот результат очень естествен с физической точки зрения, в данном формализме он выглядит фактом замечательным и нетривиальным.

Чтобы получить спектр оператора площади в интересующем нас случае, достаточно положить в выражении (41) $a=b=0$ :

$$
\mathcal{S}(\Sigma)=8 \pi \hbar G \sqrt{C(\mathrm{so}(3))-C_{1}(\mathrm{so}(3,1))} .
$$

Полученный спектр не зависит от параметра Иммирци вследствие независимости от $\beta$ коммутатора триадного мультиплета со сдвинутой связностью

$$
\left[\mathcal{A}_{i}^{X}, \widetilde{P}_{Y}^{j}\right]=i \hbar \delta_{i}^{j} I_{(P) Y}^{X} .
$$

Это позволяет надеяться, что все физические величины не будут зависеть от параметра Иммирци. Отсюда также следует, что предлагаемое квантование свободно от проблем

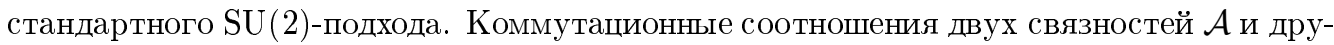
гие полезные формулы приведены в работах [12], [13]. 
5.3. Воспроизведение результатов $\mathrm{SU}(2)$-подхода. Заметим, что при выборе параметров $a$ и $b$ в уравнении (41) в виде

$$
a=-\beta, \quad b=1
$$

мы воспроизводим спектр оператора плошади (8), найденный в стандартном подходе [3]:

$$
\mathcal{S} \sim \hbar \beta \sqrt{C(\mathrm{su}(2))} .
$$

Более того, связность, используемая в этом случае, является so $(3,1)$-обобщением стандартной связности Аштекара-Барберо (2). Действительно, из (36) со значениями параметров (48) находим

$$
\mathbf{A}_{i}^{X} \equiv \mathcal{A}_{i}^{X}(-\beta, 1)=I_{(Q) Y}^{X}\left(\delta_{Z}^{Y}-\beta \Pi_{Z}^{Y}\right) A_{i}^{Z}-\beta R_{Y}^{X} \Lambda_{i}^{Y}(\chi),
$$

где $\Lambda_{i}^{X}(\chi)$ задано в (38). Эта лоренцева связность обладает следующими свойствами, являющимися прямым обобшением свойств связности Аштекара-Барберо:

1) во временной калибровке $\chi=0$ она совпадает со связностью Аштекара-Барберо

$$
\mathbf{A}_{i}^{X} \underset{\chi=0}{=}\left(0, \frac{1}{2} \varepsilon^{a}{ }_{b c} \omega_{i}^{b c}-\beta \omega_{i}^{0 a}\right)
$$

2) связность (50) коммутативна:

$$
\left\{\mathbf{A}_{i}^{X}, \mathbf{A}_{j}^{Y}\right\}_{\mathrm{D}}=0
$$

3 ) из уравнения (39) следует ковариантное обобщение соотношения (3),

$$
\left\{\mathbf{A}_{i}^{X}, \widetilde{Q}_{Y}^{j}\right\}_{\mathrm{D}}=\beta \delta_{i}^{j} I_{(Q) Y}^{X} .
$$

Таким образом, стандартные результаты $\mathrm{SU}(2)$-подхода оказываются включенными в предложенный выше формализм. Более того, они имеют лоренц-ковариантное расширение и могут быть строго выведены (включая структуру гильбертова пространства [13]) из ковариантного квантования. Благодаря этому можно сделать вывод о справедливости $\mathrm{SU}(2)$-подхода, рассмотрев статус этих результатов в ковариантном формализме.

Из предыдущего раздела ясно, что квантование, основанное на связности (50), приводит к аномалии в четырехмерной диффеоморфной инвариантности. Если бы эта аномалия была неизбежным свойством квантовой гравитации, мы бы могли говорить о нетривиальных физических следствиях. Однако, как мы видели, сушествует квантование, описываемое уравнениями (45) и (47), в котором данная проблема не возникает, и поэтому именно оно должно рассматриваться как единственно правильное в рамках петлевого подхода. Поэтому очевидно, что квантование, ведущее к результатам $\mathrm{SU}(2)$-формализма, и сам этот формализм неправильны.

Кроме того, мы приходим к естественному объяснению проблемы параметра Иммирци. Ее появление есть не что иное, как отражение нарушения диффеоморфной симметрии на квантовом уровне. Если же мы сохраняем все классические симметрии при квантовании, параметр Иммирци остается таким же нефизическим параметром, каким он 
был в классической теории. Этот результат подтверждается и анализом функционального интеграла в разделе 4. Заметим, что построенньй там функциональный интеграл не связан с каким-либо выбором связности, а опирается только на BRST-анализ классической алгебры симметрии.

Более того, ковариантный формализм показывает, что истинный произвол в квантовании связан не с параметром Иммирци, введенным в классическое действие, а с выбором связности в определении петлевых операторов. Это очевидно из коммутационных соотношений (39) и спектра оператора плошади (41). Они зависят только от параметров $a$ и $b$ из определения связности, но не от $\beta$. Зависимость же от параметра Иммирци появляется только после отождествления (48). В любом случае этот произвол полностью фиксируется требованием сохранения всех классических симметрий.

Таким образом, наш главный вывод состоит в том, что стандартное петлевое квантование, основанное на SU(2)-калибровочной группе, является неправильным подходом, а корректное квантование должно основываться на связности (45) и приводит к спектру оператора площади (46), не зависящему от параметра Иммирци.

\section{6. ЗАКЛЮЧЕНИЕ}

В этой работе мы представили обзор лоренц-ковариантного петлевого подхода к квантовой гравитации. Этот подход основан на новой гамильтоновой формулировке общей теории относительности (14) с калибровочной группой $\mathrm{SO}(3,1)$. Она строится без какой-либо фиксации калибровки и явно ковариантна относительно калибровочных преобразований. Мы применили построенный формализм для исследования зависимости квантовой гравитации от параметра Иммирци $\beta$, явно входящего в исходное действие (10). Так было показано, что функциональный интеграл не зависит от этого параметра. Далее ковариантный формализм был использован как основа для петлевого подхода к квантованию гравитации. В рамках этого подхода мы исследовали оператор площади произвольной пространственноподобной поверхности. Мы показали, что в петлевом квантовании сушествует произвол, заключаюшийся в выборе связности в определении вильсоновских линий и нашли все лоренцевы связности, диагонализующие оператор площади. Они образуют двухпараметрическое семейство. Для всех членов этого семейства вычислен соответствующий спектр площади (41).

Однако только одна связность (45) из двухпараметрического семейства преобразуется правильным образом при временны́х диффеоморфизмах и потому является пространственно-временно́й связностью. Поэтому именно она должна использоваться для квантования. Это устраняет произвол в петлевом квантовании, связанный с выбором связности в определении петлевых операторов. Соответствующий спектр оператора площади (46) не зависит от параметра Иммирци и дает ненулевое значение для минимального кванта площади.

Из полученных результатов следует, что стандартный петлевой подход, основанный на калибровочной группе $\mathrm{SU}(2)$, не может быть правильным квантованием гравитации. Это заключение есть следствие того, что вся структура SU(2)-петлевой квантовой гравитации может быть выведена из ковариантного квантования [13]. Но так как обобшение связности Аштекара-Барберо не является пространственно-временной связностью, то 
основанное на ней квантование неизбежно нарушает диффеоморфную инвариантность теории. В частности, появление зависимости физических результатов от параметра Иммирци есть отражение этого нарушения.

Эти результаты получили свое продолжение в работе [12], где в предположении, что все операторы плошади одновременно диагонализуются, было исследовано гильбертово пространство петлевой квантовой гравитации в ковариантном формализме. Показано, что сушествует базис, реализованный лоренш-ковариантными SN, которые являются собственными состояниями операторов площади любой пространственноподобной поверхности. Они строятся из лоренцевых вильсоновских линий, спроецированных на неприводимые представления $\mathrm{SO}(3)$-подгруппы. Эта проекция решает проблему бесконечномерности унитарных представлений, связанную с некомпактностью группы Лоренца, а также проблему некоммутативности лоренцевой связности. Кроме того, получено, что только простые представления группы Лоренца ассоциируются с вильсоновскими линиями. Этот результат согласуется с результатами "spin foam" моделей квантовой гравитации [25].

Главным следующим шагом в развитии теории было бы строгое доказательство того, что построенное гильбертово пространство действительно является кинематическим пространством состояний квантовой гравитации (или нахождение его недостающих компонент). Для этого прежде всего нужно решить очень непростую проблему, как наложить связи второго рода непосредственно на уровне гильбертова пространства, т.е. как некие алгебраические связи на петлевые операторы.

Среди дальнейших перспектив можно также выделить вычисление энтропии черной дыры. Для этого необходимо обобщить вывод формулы Бекенштейна-Хокинга, сделанный для $\mathrm{SU}(2)$-случая [5]. Серьезным препятствием на этом пути является непрерывность спектра площади. Каким образом оно должно преодолеваться - остается до сих пор непонятным. Но в любом случае, как и для оператора плошади, ответ не будет зависеть от параметра Иммирци.

Следуюшие естественные шаги - вычисление спектра оператора объема и (может быть, наиболее важный) определение квантового оператора гамильтоновой связи. В отличие от $\mathrm{SU}(2)$-случая в ковариантном формализме эта связь полиномиальна по каноническим переменным, и поэтому здесь не должно возникнуть проблем, появляющихся в стандартном подходе.

ПРИЛОЖЕНИЕ А

Введем матрицы, связывающие различные триадные мультиплеты,

$$
\begin{array}{ll}
\widetilde{P}_{X}^{i}=\Pi_{X}^{Y} \widetilde{Q}_{Y}^{i}, & \Pi^{X Y}=\left(\begin{array}{ll}
0 & 1 \\
1 & 0
\end{array}\right) \delta_{a}^{b}, \\
\widetilde{P}_{X}^{i}=\frac{R}{1+\beta^{-2}} \widetilde{P}_{(\beta) Y^{i}}, & R^{X Y}=\left(\begin{array}{cc}
1 & -\beta^{-1} \\
-\beta^{-1} & -1
\end{array}\right)^{-1} \delta_{a}^{b} .
\end{array}
$$


Для них выполняются следующие соотношения:

$$
\begin{aligned}
R^{X Y} & =g^{X Y}-\beta^{-1} \Pi^{X Y} \\
\left(\Pi^{-1}\right)_{X}^{Y} & =-\Pi_{X}^{Y}, \quad\left(R^{-1}\right)^{X Y}=\frac{g^{X Y}+\beta^{-1} \Pi^{X Y}}{1+\beta^{-2}} .
\end{aligned}
$$

Благодаря им матрищы П, $R$ и их обратные матрицы коммутируют друг с другом. Более того, они коммутируют со структурными константами в следующем смысле:

$$
f^{X Y Z^{\prime}} \Pi_{Z^{\prime}}^{Z}=f^{X Y^{\prime} Z} \Pi_{Y^{\prime}}^{Y}
$$

Свертка двух структурных констант может быть представлена как

$$
f_{X Y}^{T} f_{T Z}^{W}=-g_{X Z} \delta_{Y}^{W}+g_{Y Z} \delta_{X}^{W}+\Pi_{X Z} \Pi_{Y}^{W}-\Pi_{Y Z} \Pi_{X}^{W}
$$

Приведем еще несколько полезных соотношений:

$$
\begin{gathered}
\widetilde{P}_{X}^{j} \partial_{i}{\underset{\sim}{\sim}}_{j}^{X}=-\underset{\sim}{P_{j}^{X}} \partial_{i} \widetilde{Q}_{X}^{j}=0, \\
f^{W Y Z} I_{(P) W}^{X} \widetilde{Q}_{Y}^{i} \widetilde{Q}_{Z}^{j}=0, \\
f^{W Y Z} I_{(Q) W}^{X} \widetilde{Q}_{Y}^{i} \widetilde{Q}_{Z}^{j}=f^{X Y Z} \widetilde{Q}_{Y}^{i} \widetilde{Q}_{Z}^{j} .
\end{gathered}
$$

ПРИЛОЖКЕНИЕ Б

Определим усредненные связи

$$
\left.\mathcal{G}(n)=\int d^{3} x n^{X} \mathcal{G}_{X}, \quad \mathcal{D}(\vec{N})=\int d^{3} x N^{i} \mathcal{D}_{i}, \quad \underset{\sim}{\mathcal{H}} \underset{\sim}{N}\right)=\int d^{3} x \underset{\sim}{N} \mathcal{H}
$$

Они образуют следующую алгебру:

$$
\begin{aligned}
& \{\mathcal{G}(n), \mathcal{G}(m)\}_{\mathrm{D}}=\mathcal{G}(n \times m), \quad\{\mathcal{D}(\vec{N}), \mathcal{D}(\vec{M})\}_{\mathrm{D}}=-\mathcal{D}([\vec{N}, \vec{M}]), \\
& \{\mathcal{D}(\vec{N}), \mathcal{G}(n)\}_{\mathrm{D}}=-\mathcal{G}\left(N^{i} \partial_{i} n\right), \quad\{\mathcal{H}(\underset{\sim}{N}), \mathcal{G}(n)\}_{\mathrm{D}}=0, \\
& \{\mathcal{D}(\vec{N}), \mathcal{H}(\underset{\sim}{N})\}_{\mathrm{D}}=-\mathcal{H}\left(\mathcal{L}_{\vec{N}} \underset{\sim}{N}\right), \quad\{\mathcal{H}(\underset{\sim}{N}), \mathcal{H}(\underset{\sim}{M})\}_{\mathrm{D}}=\mathcal{D}(\vec{K})-\mathcal{G}\left(K^{j} A_{j}\right),
\end{aligned}
$$

где

$$
\begin{aligned}
(n \times m)^{X} & =f_{Y Z}^{X} n^{Y} m^{Z}, \quad \mathcal{L}_{\vec{N}} \underset{\sim}{N}=N^{i} \partial_{i} \underset{\sim}{N}-\underset{\sim}{N} \partial_{i} N^{i}, \\
{[\vec{N}, \vec{M}]^{i} } & =N^{k} \partial_{k} M^{i}-M^{k} \partial_{k} N^{i}, \\
K^{j} & \left.=\underset{\sim}{N \partial_{i} M} \underset{\sim}{M} \underset{\sim}{M} \partial_{i}{ }_{\sim}^{N}\right) \widetilde{Q}_{X}^{i} \widetilde{Q}_{Y}^{j} g^{X Y} .
\end{aligned}
$$

Благодарности. Автор выражает благодарность региональному бюро ЮНЕСКО по научному сотрудничеству в Европе за поддержку Международной школы физики им. В. А. Фока. 


\section{Список литературы}

[1] C. Rovelli, L. Smolin. Phys. Rev. Lett. 1988. V. 61. P. 1155; Nucl. Phys. B. 1990. V. 331. P. 80.

[2] M. Gaul, C. Rovelli. Loop quantum gravity and the meaning of diffeomorphism invariance. In: Towards Quantum Gravity. Proc. of the 35th Intern. Winter School on Theoretical Physics (Polanica, Poland, 2-11 February 1999). Lect. Notes Phys. V. 541. Ed. J. Kowalski-Glikman. Berlin: Springer, 2000. P. 277; gr-qc/9910079.

[3] C. Rovelli, L. Smolin. Nucl. Phys. B. 1995. V. 442. P. 593; A. Ashtekar, J. Lewandowski. Class. Q. Grav. 1997. V. 14. P. 55.

[4] R. Loll. Phys. Rev. Lett. 1995. V. 75. P. 3048; R. DePietri, C. Rovelli. Phys. Rev. D. 1996. V. 54. P. 2664; J. Lewandowski. Class. Q. Grav. 1997. V. 14. P. 71.

[5] C. Rovelli. Phys. Rev. Lett. 1996. V. 77. P. 3288; A. Ashtekar, J. Baez, A. Corichi, K. Krasnov. Phys. Rev. Lett. 1998. V. 80. P. 904.

[6] J. F. Barbero. Phys. Rev. D. 1994. V. 49. P. 6935.

[7] A. Ashtekar. Phys. Rev. Lett. 1986. V. 57. P. 2244; Phys. Rev. D. 1987. V. 36. P. 1587.

[8] G. Immirzi. Nucl. Phys. Proc. Suppl. 1997. V. 57. P. 65.

[9] S. Alexandrov. Class. Q. Grav. 2000. V. 17. P. 4255.

[10] S. Alexandrov, D. Vassilevich. Phys. Rev. D. 2001. V. 64. P. 044023.

[11] S. Alexandrov. Phys. Rev. D. 2002. V. 65. P. 024011.

[12] S. Alexandrov. Phys. Rev. D. 2002. V. 66. P. 024028.

[13] S. Alexandrov, E. R. Livine. Phys. Rev. D. 2003. V. 67. P. 044009; gr-qc/0209105.

[14] A. Ashtekar. Lectures on Non-Perturbative Canonical Gravity. Singapore: World Scientific, 1991.

[15] T. Thiemann. Class. Q. Grav. 1996. V. 13. P. 1383; A. Ashtekar. Phys. Rev. D. 1996. V. 53. P. 2865.

[16] A. Ashtekar, J. D. Romano, R. S. Tate. Phys. Rev. D. 1989. V. 40. P. 2572; G. Immirzi. Class. Q. Grav. 1993. V. 10. P. 2347.

[17] A. Ashtekar, J. Lewandowski. J. Math. Phys. 1995. V. 36. P. 2170; J. Geom. Phys. 1995. V. 17. P. 191.

[18] C. Rovelli, L. Smolin. Phys. Rev. D. 1995. V. 52. P. 5743; J. Baez. Spin networks in nonperturbative quantum gravity. In: The interface of knots and physics. Ed. L. Kauffman. Providence, Rhode Island: American Mathematical Society, 1996. P. 167; gr-qc/9504036.

[19] C. Rovelli, T. Thiemann. Phys. Rev. D. 1998. V. 57. P. 1009.

[20] S. Holst. Phys. Rev. D. 1996. V. 53. P. 5966.

[21] E. S. Fradkin, G. A. Vilkovisky. Phys. Lett. B. 1975. V. 55. P. 244; I. A. Batalin, G. A. Vilkovisky. Phys. Lett. B. 1977. V. 69. P. 309; E. S. Fradkin, T. E. Fradkina. Phys. Lett. B. 1978. V. 72. P. 343.

[22] M. Henneaux. Phys. Rep. 1985. V. 126. P. 1.

[23] S. Alexandrov, I. Grigentch, D. Vassilevich. Class. Q. Grav. 1998. V. 15. P. 573.

[24] S. Yu. Alexandrov, D. V. Vassilevich. Phys. Rev. D. 1998. V. 58. P. 124029.

[25] J. W. Barrett, L. Crane. Class. Q. Grav. 2000. V. 17. P. 3101; A. Perez, C. Rovelli. Phys. Rev. D. 2001. V. 63. P. 041501; V. 64. P. 064002; D. Oriti. Rep. Progr. Phys. 2001. V. 64. P. 1489. 